\section{Assertive outreach could be cost-effective}

We read with interest the findings of the UK700 Group (2000) and were particularly struck by the presentation of the results. The authors start with a premise that intensive case management was thought to be of benefit in terms of quality but was considered an expensive form of care delivery. However, their findings suggest otherwise, that intensive case management can be justified in terms of cost.

That the patients who were part of smaller case-loads did not fair better may reflect the criteria for entry to the study. Having a psychotic illness of at least 2 years with two admissions to hospital could be described as representing a fairly typical cohort of general psychiatry patients, who would not meet criteria for acceptance by most current assertive outreach teams. By being more selective and focusing on a more disabled group, our service has significantly reduced local bed usage. Many of the patients' difficulties previously centred around engagement with services and it is in this area that the team had to work hardest. We have found that it is often only after 2 years of working with patients that many begin to be able to cope with the transition to independent living and to reduce their use of resources, including hospital admissions.

At a time when community mental health teams nationwide are struggling to provide a reasonable standard of care for their patients, assertive outreach can also provide satisfaction and opportunities for learning for all team members. In our experience, the knowledge that patients are receiving an acceptable standard of care rather than just 'fire-fighting' can lead to a maintenance of staff moral - a factor which does not come cheap but which the UK700 study shows can be no more expensive.

UK 700 Group (2000) Cost-effectiveness of intensive $v$ standard case management for severe psychiatric illness. UK700 case management trial. British Journal of Psychiatry, I76, 537-543.

J.Wilson Intensive Support Team, Hellesdon Hospital, Drayton High Road, Hellesdon, Norwich NR6 5BE

J. Gardner Rehabilitation Psychiatry, Hellesdon Hospital, Drayton High Road, Hellesdon, Norwich NR6 5BE

\section{Relevant training for case managers in severe mental illness}

The UK700 Group (2000) case management trial demonstrated that there were "no clear beneficial effects on costs, clinical outcome or cost effectiveness" by reducing the case-loads of case managers working with patients with psychotic disorders. However, they went on to advise that the policy of intensive case management for patients with severe psychosis is not justified. The findings of this study do not support such a conclusion. The key issue studied (i.e. intensive case management) was determined purely by numbers of patients on the manager's case-load. No attempt is made to describe the level of training of the case managers in techniques and skills needed for effective working with patients who have a severe mental illness. The need for relevant training for case managers in severe mental illness has been addressed in the Clinical Standards Advisory Group report on schizophrenia (Gournay \& Beadsmore, 1995). An alternative explanation for the findings of this study may be that without specific training in severe mental illness, purely reducing case manager's case-loads is not effective. It is most disappointing and surprising that this issue has neither been addressed in a study of this magnitude nor referred to in the discussion.

Gournay, K. \& Beadsmore, A. (1995) The report of the Clinical Standards Advisory Group: standards of care for people with schizophrenia in the UK and implications for mental health nursing. Journal of Psychiatric and Mental Health Nursing, 2, 359-364.

UK700 Group (2000) Cost-effectiveness of intensive standard case management for severe psychotic illness. UK700 case management trial. British Journal of Psychiatry, I76, 537-543.

F. M. Martin School of Nursing and Midwifery, The Queen's University of Belfast, Medical Biology Centre, 97 Lisburn Road, Belfast BT9 7BL

Authors' reply: The training and expertise of the case managers involved in the UK700 case management trial have been described in a previous publication (UK700 Group, 1999) and discussed in subsequent correspondence (Burns et al, 1999). The clinical staff providing both intensive and standard case management were equally experienced in terms of training and skills, had considerable expertise in working with severe mental illness, and were representative of the staff working in community mental health teams throughout the UK. All staff received courses in case management, and intensive case managers additionally received training in outreach practice from a team leader in assertive community treatment.

The recommendations made by Gournay \& Beadsmore (1995), such as implementation of the Care Programme Approach and emphasis on post-qualification training, are ones we would agree with, and were in place in the collaborating sites during the period of the trial. They are, however, general recommendations and there is currently no research or strong clinical consensus to suggest which specific skills or training components are required for effective or costeffective care in this area.

Although we agree that the skills of case managers working with people who have severe mental illness are an important issue in need of research, Martin has misunderstood the purpose of the UK700 case management trial. We aimed to test rigorously one component of intensive case management - reduced case-load size - in a pragmatic situation representative of clinical services within the UK. We stand by the conclusions of our paper, which relate to the cost-effectiveness of low case-load size within the framework of current clinical expertise in the UK.

Burns, T., Fahy, T., Thompson, S., et al (1999) Intensive case management for severe psychotic illness (authors' reply). Lancet, 354, 1384-1386.

Gournay, K. \& Beadsmore, A. (1995) The report of the Clinical Standards Advisory Group: standards of care for people with schizophrenia in the UK and implications for mental health nursing. Journal of Psychiatric and Mental Health Nursing, 2, 359-364.

UK700 Group (1999) Intensive versus standard case management for severe psychotic illness: a randomised trial. Lancet, 353, 2185-2189.

S. Byford Centre for Health Economics, University of York, Heslington, York YOI 5DD

M. Fiander, T. Burns St George's Hospital Medical School, Cranmer Terrace, Tooting, London SWI7 ORE

T. Fahy South London \& Maudsley NHS Trust, Denmark Hill, London SE5 8AZ

\section{Neurocognitive deficits in infants of mothers with schizophrenia}

Yoshida et al (1999) demonstrate the need for further longitudinal observational studies of the mother-infant relationship when mothers have schizophrenia that 
began during pregnancy. It is important to disentangle the impact on the infant of the mother-infant relationship and the environment she provides for her child from those genetic factors which place the infant at risk.

Neurobiological features are suggested by reports that: variations in maternal care in the rat promote hippocampal synaptogenesis and spatial learning and memory through systems known to mediate experience-dependent neural development (Liu et al, 2000); schizophrenia is a disorder of developmentally reduced synaptic connectivity (McGlashan \& Hoffman, 2000); and selective deficits in early-stage sensory processing in schizophrenia are due to a failure to support the entrainment of intrinsic gamma-frequency oscillations $(30-50 \mathrm{~Hz}$ or broader, centred on $40 \mathrm{~Hz}$ ) (Friedman \& Coats, 2000) involved in processes associated with encoding into sensory memory both at the cellular level (synaptic potentiation) and at the cognitive level (Haenschel et al, 2000).

This hypothesis is supported by shortterm laboratory experience demonstrating that adult female speech production is sufficient to influence infant speech production occurring in the silent intervals between the adult vocalisations of the order of 3 seconds. This is linked with increased coherence of electroencephalograph gammaband activity associated with the execution of more complex tasks (Friedman \& Coats, 2000); language discrimination by human newborns may be influenced by hearing rhythmic aspects of speech while in the womb, a period in development during which exposure may have a more profound impact on the organisation of the brain than does learning after birth (Ramus et al, 2000).

These findings prompt the possibility of prevention of neurocognitive defects (at least those of a sensory and perceptual nature) by establishing effective cortical oscillations, starting during pregnancy as suggested by Yoshida $e t$ al.

Friedman, E. H. \& Coats, A. J. S. (2000)

Neurobiology of exaggerated heart rate oscillations during two meditative techniques (letter). International Journal of Cardiology, 73, 199

Haenschel, C., Baldeweg, T., Croft, R. J., et al (2000) Gamma and beta frequency oscillations in response to novel auditory stimuli: A comparison of human electroencephalogram (EEG) data with in vitro models. Proceedings of the National Academy of Sciences of the United States of America, 97, 7645-7650.
Liu, D., Diorio, J., Francis, D. D., et al (2000) Maternal care, hippocampal synaptogenesis and cognitive development in rats. Nature Neuroscience, 3, 799-806.

\section{McGlashan, T. H. \& Hoffman, R. E. (2000)}

Schizophrenia as a disorder of developmentally reduced synaptic connectivity. Archives of General Psychiatry, 57 637-648.

Ramus, F., Hauser, M. D., Miller, C., et al (2000) Language discrimination by human newborns and by cotton-top tamarin monkeys. Science, 288, 349-35I.

Yoshida, K., Marks, M. N., Craggs, M., et al (1999) Sensorimotor and cognitive development of infants of mothers with schizophrenia. British Journal of Psychiatry 175, 380-387.

E. H. Friedman I83| Forest Hills Boulevard, Cleveland, $\mathrm{OH} 44 \mathrm{II}$, USA

\section{Disclosing the diagnosis of dementia}

We read with interest the paper about truth-telling and the diagnosis of dementia (Pinner, 2000). The thrust of the article is that people with dementia should be told the diagnosis in the same way that patients are told that they are suffering from cancer. The disadvantages of this approach are stated but underplayed. In clinical practice it is common to see patients who have been told the diagnosis of cancer, sometimes with such frankness that they have gone on to develop major psychological sequelae and sometimes fatal decline.

We experienced this recently when a 58-year-old woman, after being made aware of her diagnosis of dementia, developed severe depression and suicidal ideas. The depression worsened her cognitive state and made her non-compliant to intervention. Cognitive decline makes patients more vulnerable and reduces their ability to cope with stress (Clafferty, 1999). Suicides after disclosure of diagnosis have been described (Rohde et al, 1995). Insight regarding progressive cognitive decline is an important determinant of reaction to disclosure. In insightful patients the risk of depressive reactions and suicide must be seriously considered after disclosure of any major illness (Maguire et al, 1996).

The debate about this issue is a further example of the importance of dealing with each patient as an individual. It is good practice for every patient to be informed about the illness and its implications. It is equally important to accept that some patients do not want to know the nature of their illness and informing them is harmful. This perspective needs greater emphasis in a climate when telling everyone is sometimes seen as the only approach.

Clafferty, R. A. (1999) Dignity in knowledge. Informing patients with Alzheimer's disease about their diagnosis. Psychiatric Bulletin, 23, 394-396.

Maguire, C. P., Kirby, M., Coen, R., et al (1996) Family members' attitudes towards telling the patient with Alzheimer's disease their diagnosis. British Medical Journal, 313, 529-530.

Pinner, G. (2000) Truth-telling and the diagnosis of dementia. British journal of Psychiatry, 176, 514-515.

Rohde, K., Peskind, E. R. \& Raskind, M. A. (1995)

Suicide in two patients with Alzheimer's disease. Journal of the American Geriatric Society, 43, 187-189.

A. Ahujn, D. D. R. Williams Cefn Coed Hospital, Waunarlwydd Road, Cockett, Swansea SA2 $0 \mathrm{GH}$

\section{Need for neuropathological studies in pre-senile dementia}

Kay et al's paper (2000) on long-term survival in pre-senile dementia adds a useful and important contribution to this underresearched area. While acknowledging the difficulties faced in drawing valid conclusions from a non-neuropathologically confirmed study, there are several points of interest and concern not raised by the authors.

Pre-senile dementia is a heterogeneous group of disorders and the report that only 19 of 233 cases were not pre-senile dementia of Alzheimer type or pre-senile vascular dementia is a concern. The authors previously recognised that cases defined as Alzheimer's disease by clinical criteria alone may include conditions with non-Alzheimer type pathology, such as Pick's disease (Newens et al, 1993), but felt this reflected only a small number of patients. However, recent evidence suggests that the frontotemporal dementia (FTD) may account for up to a quarter of patients presenting before the age of 65 (Snowden et al, 1996). Retrospective analysis of case notes using the NINCDS-ADRDA criteria (McKhann et al, 1984) for diagnosing Alzheimer's disease may well include many FTDs, as the criteria for a diagnosis of probable Alzheimer's disease are also features of this subgroup.

The diagnosis was reportedly confirmed in a proportion as part of a case-control study, although there is a risk of selection bias by possible exclusion of the more behaviourally challenging uncooperative FTD patients. 